\title{
Reactive Transport Modeling of Column Experiments for the Remediation of Acid Mine Drainage
}

Richard T. Amos, ${ }^{*} \dagger$ K. Ulrich Mayer, ${ }^{\dagger}$ David W. Blowes, ${ }^{\ddagger}$ and Carol J. Ptacek ${ }^{\ddagger}, \S$

${ }^{\dagger}$ Dept. Earth and Ocean Sciences, Univ. British Columbia, Vancouver, BC, V6T 1Z4, Canada

Dept. Earth Sciences, Univ. Waterloo, Waterloo, ON, N2L 3G1, Canada

and

${ }^{\S}$ National Water Research Institute, Environment Canada, Burlington, ON, L7R 4A6, Canada

* Corresponding author phone: (604)822-6080; fax (604)822-6088; email; ramos@eos.ubc.ca

\section{Supporting Information}

Contains 2 pages;

Table S1: Aqueous complexes and hydrolysis products, stoichiometric coefficients and equilibrium constants used in simulations of columns 1 and 2 . 


\begin{tabular}{|c|c|c|c|c|c|c|c|c|c|c|c|c|c|c|}
\hline Species & \multicolumn{13}{|c|}{ Components } & \multirow{2}{*}{$\log K$} \\
\hline & $\mathrm{Ca}$ & $\mathrm{CO}_{3}$ & $\mathrm{Cl}$ & $\mathrm{Fe}(\mathrm{II})$ & $\mathrm{H}^{+}$ & HS & $\mathrm{K}$ & $\mathrm{Mg}$ & $\mathrm{Mn}$ & $\mathrm{Na}$ & $\mathrm{Ni}$ & $\mathrm{SO}_{4}$ & $\mathrm{Zn}$ & \\
\hline $\mathrm{OH}^{-}$ & & & & & -1 & & & & & & & & & $\begin{array}{l}- \\
13.998\end{array}$ \\
\hline $\mathrm{Mg}(\mathrm{OH})^{+}$ & & & & & -1 & & & 1 & & & & & & $-\overline{11.440}$ \\
\hline $\mathrm{MgCO}_{3}(\mathrm{aq})$ & & 1 & & & & & & 1 & & & & & & 2.9800 \\
\hline $\mathrm{MgHCO}_{3}^{+}$ & & 1 & & & 1 & & & 1 & & & & & & 11.400 \\
\hline $\mathrm{MgSO}_{4}(\mathrm{aq})$ & & & & & & & & 1 & & & & 1 & & 2.3700 \\
\hline $\mathrm{Ca}(\mathrm{OH})^{+}$ & 1 & & & & -1 & & & & & & & & & $-\overline{12.780}$ \\
\hline $\mathrm{CaHCO}_{3}^{+}$ & 1 & 1 & & & 1 & & & & & & & & & 11.440 \\
\hline $\mathrm{CaCO}_{3}(\mathrm{aq})$ & 1 & 1 & & & & & & & & & & & & 3.2200 \\
\hline $\mathrm{CaSO}_{4}(\mathrm{aq})$ & 1 & & & & & & & & & & & 1 & & 2.3090 \\
\hline $\mathrm{CaHSO}_{4}^{+}$ & 1 & & & & 1 & & & & & & & 1 & & 3.0680 \\
\hline $\mathrm{NaCO}_{3}^{-}$ & & 1 & & & & & & & & 1 & & & & 1.2680 \\
\hline $\mathrm{NaHCO}_{3}(\mathrm{aq})$ & & 1 & & & 1 & & & & & 1 & & & & 10.080 \\
\hline $\mathrm{NaSO}_{4}^{-}$ & & & & & & & & & & 1 & & 1 & & 0.7000 \\
\hline $\mathrm{KSO}_{4}^{-}$ & & & & & & & 1 & & & & & 1 & & 0.8500 \\
\hline $\mathrm{Fe}(\mathrm{OH})^{+}$ & & & & 1 & -1 & & & & & & & & & 9.5000 \\
\hline $\mathrm{Fe}(\mathrm{OH})_{3}^{-}$ & & & & 1 & -3 & & & & & & & & & $\begin{array}{l}- \\
31.000\end{array}$ \\
\hline $\mathrm{FeSO}_{4}(\mathrm{aq})$ & & & & 1 & & & & & & & & 1 & & 2.2500 \\
\hline $\mathrm{FeHSO}_{4}^{+}$ & & & & 1 & 1 & & & & & & & 1 & & 3.0680 \\
\hline $\mathrm{FeHCO}_{3}^{+}$ & & 1 & & 1 & 1 & & & & & & & & & 12.330 \\
\hline $\mathrm{FeCO}_{3}(\mathrm{aq})$ & & 1 & & 1 & & & & & & & & & & 4.3800 \\
\hline $\mathrm{Fe}(\mathrm{OH})_{2}(\mathrm{aq})$ & & & & 1 & -2 & & & & & & & & & $\begin{array}{l}- \\
20.570\end{array}$ \\
\hline $\mathrm{Fe}(\mathrm{HS})_{2}(\mathrm{aq})$ & & & & 1 & & 2 & & & & & & & & 8.9500 \\
\hline $\mathrm{Fe}(\mathrm{HS})_{3}$ & & & & 1 & & 3 & & & & & & & & 10.987 \\
\hline $\mathrm{FeCl}^{+}$ & & & 1 & 1 & & & & & & & & & & 0.1400 \\
\hline $\mathrm{MnCl}^{+}$ & & & 1 & & & & & & 1 & & & & & 0.6070 \\
\hline $\mathrm{MnCl}_{2}(\mathrm{aq})$ & & & 2 & & & & & & 1 & & & & & 0.2500 \\
\hline $\mathrm{MnCl}_{3}^{-}$ & & & 3 & & & & & & 1 & & & & & $\overline{-}-3050$ \\
\hline $\mathrm{Mn}(\mathrm{OH})^{+}$ & & & & & -1 & & & & 1 & & & & & $\begin{array}{l}- \\
10.590\end{array}$ \\
\hline $\mathrm{Mn}(\mathrm{OH})_{3}^{-}$ & & & & & -3 & & & & 1 & & & & & $-\overline{34.800}$ \\
\hline $\mathrm{MnCO}_{3}(\mathrm{aq})$ & & 1 & & & & & & & 1 & & & & & 4.9000 \\
\hline $\mathrm{MnSO}_{4}(\mathrm{aq})$ & & & & & & & & & 1 & & & 1 & & 2.2600 \\
\hline $\mathrm{MnHCO}_{3}^{+}$ & & 1 & & & 1 & & & & 1 & & & & & 12.280 \\
\hline $\mathrm{ZnCl}^{+}$ & & 1 & & & & & & & & & & & 1 & 0.4300 \\
\hline $\mathrm{ZnCl}_{(}(\mathrm{aq})$ & & 2 & & & & & & & & & & & 1 & 0.4500 \\
\hline $\mathrm{ZnCl}_{3}^{-}$ & & 3 & & & & & & & & & & & 1 & 0.5000 \\
\hline
\end{tabular}




\begin{tabular}{|c|c|c|c|c|c|c|c|c|c|c|c|c|c|c|}
\hline \multirow{2}{*}{ Species } & \multicolumn{13}{|c|}{ Components } & \multirow{2}{*}{$\log K$} \\
\hline & $\mathrm{Br}$ & $\mathrm{CO}_{3}$ & $\mathrm{Cl}$ & $\mathrm{Fe}(\mathrm{II})$ & $\mathrm{H}^{+}$ & HS & $\mathrm{K}$ & $\mathrm{Mg}$ & $\mathrm{Mn}$ & $\mathrm{Na}$ & $\mathrm{Ni}$ & $\mathrm{SO}_{4}$ & $\mathrm{Zn}$ & \\
\hline $\mathrm{ZnCl}_{4}^{2-2}$ & & & 4 & & & & & & & & & & 1 & 0.1990 \\
\hline $\mathrm{Zn}(\mathrm{OH})^{+}$ & & & & & -1 & & & & & & & & 1 & 8.9600 \\
\hline $\mathrm{Zn}(\mathrm{OH})_{2}(\mathrm{aq})$ & & & & & -2 & & & & & & & & 1 & $-\overline{16.899}$ \\
\hline $\mathrm{Zn}(\mathrm{OH})_{3}^{-}$ & & & & & -3 & & & & & & & & 1 & $\begin{array}{l} \\
28.399\end{array}$ \\
\hline $\mathrm{Zn}(\mathrm{OH})_{4}^{2-}$ & & & & & -4 & & & & & & & & 1 & -41.199 \\
\hline $\mathrm{Zn}(\mathrm{OH}) \mathrm{Cl}(\mathrm{aq})$ & & & 1 & & -1 & & & & & & & & 1 & $\begin{array}{l} \\
7.4800\end{array}$ \\
\hline $\mathrm{Zn}(\mathrm{HS})_{,}(\mathrm{aq})$ & & & & & & 2 & & & & & & & 1 & 14.940 \\
\hline $\mathrm{Zn}(\mathrm{HS})_{3}$ & & & & & & 3 & & & & & & & 1 & 16.100 \\
\hline $\mathrm{ZnSO}_{4}(\mathrm{aq})$ & & & & & & & & & & & & 1 & 1 & 2.3700 \\
\hline $\mathrm{Zn}\left(\mathrm{SO}_{4}\right)_{2}^{2-}$ & & & & & & & & & & & & 2 & 1 & 3.2800 \\
\hline $\mathrm{ZnBr}^{+}$ & 1 & & & & & & & & & & & & 1 & $-\overline{0.5800}$ \\
\hline $\mathrm{ZnBr}_{2}(\mathrm{aq})$ & 2 & & & & & & & & & & & & 1 & $-\overline{0.9800}$ \\
\hline $\mathrm{ZnHCO}_{3}^{+}$ & & 1 & & & 1 & & & & & & & & 1 & 12.400 \\
\hline $\mathrm{ZnCO}_{3}(\mathrm{aq})$ & & 1 & & & & & & & & & & & 1 & 5.3000 \\
\hline $\mathrm{Zn}\left(\mathrm{CO}_{3}\right)_{2}^{2-}$ & & 2 & & & & & & & & & & & 1 & 9.6300 \\
\hline $\mathrm{NiBr}^{+}$ & 1 & & & & & & & & & & 1 & & & 0.5000 \\
\hline $\mathrm{NiCl}^{+}$ & & & 1 & & & & & & & & 1 & & & 0.3990 \\
\hline $\mathrm{Ni}(\mathrm{OH})^{+}$ & & & & & -1 & & & & & & 1 & & & 9.8600 \\
\hline $\mathrm{Ni}(\mathrm{OH})_{2}(\mathrm{aq})$ & & & & & -2 & & & & & & 1 & & & $-\overline{19.000}$ \\
\hline $\mathrm{Ni}(\mathrm{OH})_{3}^{-}$ & & & & & -3 & & & & & & 1 & & & $\begin{array}{l}- \\
30.000\end{array}$ \\
\hline $\mathrm{NiSO}_{4}(\mathrm{aq})$ & & & & & & & & & & & 1 & 1 & & 2.2900 \\
\hline $\mathrm{NiCl}_{2}(\mathrm{aq})$ & & & 1 & & & & & & & & 1 & & & 0.9600 \\
\hline $\mathrm{NiHCO}_{3}^{+}$ & & 1 & & & 1 & & & & & & 1 & & & 12.470 \\
\hline $\mathrm{NiCO}_{3}(\mathrm{aq})$ & & 1 & & & & & & & & & 1 & & & 6.8700 \\
\hline $\mathrm{Ni}\left(\mathrm{CO}_{3}\right)_{2}^{2-}$ & & 2 & & & & & & & & & 1 & & & 10.110 \\
\hline $\mathrm{Ni}\left(\mathrm{SO}_{4}\right)_{2}^{2-2}$ & & & & & & & & & & & 1 & 2 & & 1.0200 \\
\hline $\mathrm{HCO}_{3}^{-}$ & & 1 & & & 1 & & & & & & & & & 10.330 \\
\hline $\mathrm{H}_{2} \mathrm{CO}_{3}(\mathrm{aq})$ & & 1 & & & 2 & & & & & & & & & 16.681 \\
\hline $\mathrm{HSO}_{4}^{-}$ & & & & & 1 & & & & & & & 1 & & 1.9870 \\
\hline $\mathrm{H}_{2} \mathrm{~S}(\mathrm{aq})$ & & & & & 1 & 1 & & & & & & & & 6.9940 \\
\hline $\mathrm{S}^{2-2}$ & & & & & -1 & 1 & & & & & & & & $-\overline{12.918}$ \\
\hline
\end{tabular}


Table S1. Aqueous complexes and hydrolysis products, stoichiometric coefficients and equilibrium constants used in simulations of columns 1 and 2. Equilibrium constants are from the WATEQ4F (19) and MINTEQA2 (20)

databases. 